\title{
The effect of Plantago major seed mucilage combined with carbopol on the release profile and bioadhesive properties of propranolol $\mathrm{HCl}$ buccoadhesive tablets
}

\author{
Jafar Akbari ${ }^{1,2}$, Majid Saeedi ${ }^{1,2^{*}}$, Katayoun Morteza-Semnani, ${ }^{2,3}$, Behnaz Zarrabi ${ }^{1}$, Seyyed \\ Sohrab Rostamkalaei ${ }^{4}$, Hamid Reza Kelidari ${ }^{2}$
}

${ }^{1}$ Department of Pharmaceutics, Faculty of Pharmacy, Mazandaran University of Medical Science, Sari, Iran

${ }^{2}$ Pharmaceutical Sciences Research Center, Mazandaran University of Medical Science, Sari, Iran

${ }^{3}$ Department of Medicinal Chemistry, Faculty of Pharmacy, Mazandaran University of Medical Science, Sari, Iran

${ }^{4}$ Student Research Committee, Mazandaran University of Medical Science, Sari, Iran

\begin{abstract}
Buccoadhesive drug delivery systems have distinct advantages in comparison with oral administration. Plant exudates like gum or mucilage are being studied for their use as pharmaceutical adjuvant. The aim of this study is to evaluate the properties of the Plantago major seed mucilage as a mucoadhesive agent and propranolol hydrochloride is chosen as a model drug. Mucoadhesive tablets of propranolol hydrochloride were formulated by combination of two mucoadhesive polymers include Carbopol 934P and Plantago major mucilage, and properties such as in vitro drug release, swelling, erosion, mucoadhesive force were studied. The results show increase in bioadhesive strength and decrease in release rate with increase in percent of Carbopol 934P, as F13 (containing Carbopol 934P alone) and F8 (containing mucilage alone) show the highest bioadhesive strength and highest release rate respectively and these results were matched to swelling results which decrease in swelling of matrices results in decrease in bioadhesion. Matrices with both Plantago major mucilage and Carbopol have the optimum drug release in bioadhesive formulation of propranolol tablets.
\end{abstract}

Keywords: Plantago major, mucilage, mucoadhesive, DSC, FTIR

Pharm Biomed Res 2016; 2(2): 84-100 DOI: 10.18869/acadpub.pbr.2.2.84

\section{Introduction}

The development of mucoadhesive polymers may be traced back to 1947 , when gum tragacanth and dental adhesive powders were combined to form a vehicle for applying penicillin to oral mucosa. An improvement in this system resulted when $\mathrm{CMC}$ and petrolatum were combined to form the vehicle (1). In recent years, much interest has been shown in the development of bioadhesive systems. These dosage forms can be administered by different routes such as ocular, buccal, nasal, rectal, vaginal) either for local therapy or for systemic drug delivery. In particular, the buccal route appears to offer a series of advantages compared with other routes, such as rapid onset of action, high blood supply, avoidance of first pass effect and exposure of drug to gastrointestinal tract (2). Buccal drug delivery necessitates the use of 
mucoadhesive polymers as a means of prolonging the resistance time of the dosage form on the absorbing membrane as well as localizing drugs in a particular region (3). Mucoadhesion is provided by the formation of non-covalent bonds such as hydrogen bonds and ionic interactions or physical entanglements between the mucus gel layer and polymers (4). Typical polymers that have been used as mucoadhesive drug carriers are poly acrylic acid, poly meth acrylic acid, cellulose derivatives, poly ethylene oxide, lectin and chitosan, PAA and its cross-linked commercial forms (5). By active using of copolymers, polymer conjugates and inter polymer complexes, the mucoadhesive properties of polymers have been improved. The following characteristics are the basic requirements needed for a successful buccal delivery: a bio-adhesive to retain the drug in oral cavity and maximize the intimacy of contact with mucosa, a vehicle that releases the drugs at an appropriate rate under the conditions prevailing in the mouth and strategies for overcoming the low permeability of the oral mucosa. the bioadhesive bonds are formed by three steps: wetting and swelling of mucoadhesive polymer, entanglement of polymer and mucin chains, and formation of weak chemical bonds between entangled chains and polymer (6). Mucilage and gums are well known since ancient times for their medicinal use. Today the gums are being used as pharmaceutical excipients. Mucilage generally are carbohydrate polymer which obtained from woody and non woody plant parts such as bark, seeds, sap, roots, rhizomes, fruits, and leaves. Mucilage are used for their binding, thickening, stabilizing, humidifying properties, disintegrating and release controlling in medicines $(7,8)$. Mucilage from natural sources hydrate and swells on contact with water and has been used for the preparation of single unit dosage forms (9). Plantago major is a member of Plantaginaceae family (10). The plant produces large amount of seeds. The seeds are located in capsules (816 per capsule) and become sticky in humid condition due to swelling of the polysaccharides present in the seed coat. The seeds contain the monosaccharides, disaccharides and trisaccharides. The outer seed coat, contain carbohydrate polymers that swell in contact with water and form mucilage with high viscosity. These polysaccharides are composed of xylose, arabinose, galactorunic acid, glucuronic acid, rhamnose, galactose and glucose (11). In this work, the Plantago major seed mucilage characteristics such as mucoadhesive strength, swelling, erosion and drug release rate in addition to carbopol 934P (a muccoadhesive polymer) were studied.

\section{Materials and methods}

Propranolol Hydrochloride powder, $\mathrm{HCl}$, Potassium di-hydrogen phosphate were obtained from Merck, Germany. Carbopol 934P (B.F. Goodrich, USA), Plantago major seed was purchased from herbal drug market (Sari, Iran) and $\mathrm{NaOH}$ from Dr. Mojallali (Iran). Mg stearate was obtained from Roche (Germany).

\section{Extraction of mucilage}

$10 \mathrm{~mL}$ of $0.1 \mathrm{~N} \mathrm{HCl}$ was heated to boil in a $100 \mathrm{~mL}$ corning flask. The flask was removed 
from the flame and $1 \mathrm{~g}$ test sample of dry seed was added to it. Heating resumed and the process of dissolution of the seed husk was observed. When all seeds had changed color the flask was finally removed from the flame and the solution (while being hot) was filtered through clean muslin cloth. The seeds were washed up to three times in $5 \mathrm{ml}$ of hot water and the solution obtained each time was filtered. The combined filtrate, containing the dissolved mucilage was mixed with $60 \mathrm{~mL}$ of $95 \%$ ethyl alcohol, stirred and allowed to stand for 5h. Finally the supernatant liquid was decanted off and the beaker containing the precipitate was dried an over maintained at $50{ }^{\circ} \mathrm{C}$ for 10 hours (8).

\section{Preparation of mucoadhesive Matrix Tablets}

For preparation of mucoadhesive tablets a 9 $\mathrm{mm}$ flat faced punches on a hydraulic press were used. The materials for each tablet (Table 1) were weighed and were sufficiently blended in a planetary mixer (Erweka, Germany) for 10min. magnesium stearate $(1 \% \mathrm{w} / \mathrm{w})$ was then added, followed by further mixing for $2 \mathrm{~min}$. mixed and introduced into the die and compacted at compression using $1 \%(\mathrm{M} / \mathrm{M})$ magnesium stearate. The tablet properties (crushing, strength, friability, mass variation and content uniformity) were determined by current procedure $(13,14)$.

Table1 Formulation comparison of investigated propranolol hydrochloride mucoadhesive matrix tablets

\begin{tabular}{ccccc}
\hline $\begin{array}{c}\text { Formulation } \\
\text { code }\end{array}$ & $\begin{array}{c}\text { Propranolol HCl } \\
(\mathrm{mg})\end{array}$ & $\begin{array}{c}\text { P. major } \\
\text { mucilage }(\mathrm{mg})\end{array}$ & $\begin{array}{c}\text { Carbopol 934P } \\
(\mathrm{mg})\end{array}$ & $\begin{array}{c}\text { Mg stearate } \\
(\mathrm{mg})\end{array}$ \\
\hline F1 & 80 & 10 & 70 & 1.6 \\
F2 & 80 & 20 & 60 & 1.6 \\
F3 & 80 & 30 & 50 & 1.6 \\
F4 & 80 & 40 & 40 & 1.6 \\
F5 & 80 & 50 & 30 & 1.6 \\
F6 & 80 & 60 & 20 & 1.6 \\
F7 & 80 & 70 & 10 & 1.6 \\
F8 & 80 & 40 & - & 1.2 \\
F9 & 80 & 80 & - & 2.4 \\
F10 & 80 & 160 & - & 1.2 \\
F11 & 80 & - & 40 & 1.6 \\
F12 & 80 & - & 80 & 1.6 \\
F13 & 80 & - & 160 & 2.4 \\
F14 & - & 160 & - & 1.6 \\
F15 & - & - & 160 & 1.6 \\
\hline
\end{tabular}




\section{In vitro drug release studies}

The dissolution tests were performed to the basket method (USP pharmacopeia 24). A dissolution apparatus (Caleva 8ST, Germany) was employed with a stirring rate of $100 \mathrm{rpm}$. The dissolution medium was $900 \mathrm{~mL}$ phosphate buffer (pH 6.8). Samples of the solution were withdrawn at benefit time intervals. The dissolution media was replaced by fresh dissolution fluid to maintain a constant volume. The solution was passed through a filter (Watman, USA) and then concentration of propranolol hydrochloride in solution was measured with an ultraviolet spectrophotometer (Varian, Australia) at a wavelength of $289 \mathrm{~nm}$ after suitable dilution with the dissolution medium when necessary $(n=3)$.

\section{Evaluation of tablets}

Properties include friability crushing strength, mass variation of tablets were determined by standard procedure (13). The content uniformity of active ingredient was confirmed based on British pharmacopoeia method (8). The tensile strength (T) of tablets (measure of the stress necessary to cause diametric fracture of the compact) was determined from the mean data obtained from the hardness test that we did on tablets ( $n=10)$ Using the Erweka hardness tester (TBH30MD, Germany). The T values were computed from the following equation:

$\mathrm{T}=2 \mathrm{p} / \pi^{*} \mathrm{D}_{\mathrm{t}}$

P: Load applied on the tablet that causes diametric fracture of the tablet that causes diametric fracture of the tablet

$\mathrm{D}_{\mathrm{t}}$ : Thickness of tablet (m)

\section{Drug release kinetics}

Various mathematical equations were tested for describing the kinetics of drug release from mucoadhesive tablets (zero-order, first order, Higuchi)

$$
\begin{aligned}
& \mathrm{Q}_{\mathrm{t}=} \mathrm{K}_{0} \cdot \mathrm{t} \\
& \ln Q_{t=} \ln Q_{0-} K_{1 t} \\
& Q_{t}=K_{H} t^{1 / 2}
\end{aligned}
$$

$Q_{t}$ : The amount of drug released in time $\mathrm{t}$

$Q_{0}$ : The initial amount of drug in tablet

$\mathrm{K}_{0} \cdot \mathrm{K}_{1} \mathrm{~K}_{\mathrm{H}}$ : Release rate constant for zero order, first order, Higuchi model, respectively

In order to define a model which will represent a better fit for the formulations, dissolution data can be further analyzed by Peppas and Korsemayer equation.

$\frac{M_{t}}{M_{\infty}}=K_{p} t^{n}$

\section{$\mathrm{M}_{\mathrm{t}}$ : Amount of drug released in time $\mathrm{t}$}

$\mathrm{M}_{\infty}$ : Total amount of drug that must be released at infinite time

$\mathrm{K}_{\mathrm{p}}$ : Constant

$\mathrm{n}$ : release exponent indicating the type of drug release mechanism

$\mathrm{n}$ : for a cylinder is $<0.45$ for Fickian release $>0.45$ and $<0.89$ for non- Fickian

0.89 for the case 2 release

$>0.89$ for super case 2 type release (15).

Criteria for selecting the most appropriate model were based on the best goodness of fit and smallest sum of squared residuals.

\section{Determination of bioadhesive strength}

For this purpose a tensile tester apparatus was designed similar to a tensile tester apparatus (Instron model 4301) and the 
bioadhesive strength of tablets was measured according to previously published method by a tensile tester apparatus. After isolation of hairless abdominal skin of the rat the dorsal section (mucosa part) was fixed on the head of diffusion cell and filled with phosphate buffer with $\mathrm{pH}$ 6.8. The same conditions were used exactly according to previously published method (16).

\section{Matrix swelling studies}

The study was carried out in the USP/NF dissolution apparatus No.1 (Caleva 8ST, Germany). the dry polymer matrices were accurately weighted, placed in dissolution baskets, and immersed in $900 \mathrm{~mL}$ phosphate buffer ( $\mathrm{pH}$ 6.8) maintained at $37^{\circ} \mathrm{C}$ in the dissolution vessels. At regular intervals, the pre-weighed basket-matrix system was withdrawn from the dissolution vessel, lightly bottled with a tissue paper to remove exes test liquid and re-weighed. The percent water uptake (degree of swelling due to absorbed liquid) was estimated in certain times according to the following equation:

$$
\% W U=\frac{W_{s}-W_{i}}{W_{i}} * 100
$$

$\% \mathrm{WU}$ : The percent of water uptake $(\mathrm{n}=3)$. $W_{s}$ : The weight of swelled matrix in certain time $(\mathrm{n}=3)$.

$W_{i}$ : The initial starting weight in the matrix tablet $(\mathrm{n}=3)$.

\section{Matrix erosion studies}

The standard USP/NF dissolution apparatus No.1 (Caleva8ST, Germany) was used for this purpose, the dry matrix was weighed, placed in dissolution baskets, and subjected to dissolution in $500 \mathrm{~mL}$ of $0.05 \mathrm{M}$ phosphate buffer ( $\mathrm{pH}$ 6.8) maintained at $37^{\circ} \mathrm{C}$ with the basket rotating at $100 \mathrm{rpm}$. At regular intervals basket-matrix assemblies were removed from the dissolution vessels and dried to a constant weight in a hot air oven at $60^{\circ} \mathrm{C}(17)$.

The percentage of matrix erosion $(\% \mathrm{E})$ at time, $(t)$ was estimated from the following equation:

$\% E=\frac{W_{i-} W_{t}}{W_{i}} * 100$

$W_{i}$ : The initial starting weight of the matrix

$W_{t}$ : The weight of matrix subjected to erosion for time $\mathrm{t}$

And the percentage of remaining matrix, compute from the Eq. (8)

$\%$ Remaining $=100-\% \mathrm{E}$

Eq.

(8) $(\mathrm{n}=3)$

\section{Statistical analysis}

A one-way ANOVA with a turkey's post hoc (SPSS 12), test was analyzed the dissolution and bio adhesive data obtained for each batch of formulation in order to compare the rate of drug release from the mucoadhesive tablets. The confidence limit was set at $95 \%$.

\section{Results}

The swelling factor of $1 \mathrm{~g}$ of $P$. major was $14.3 \pm 1.06 \mathrm{~mL}(637.2 \pm 43.9 \%)$ according to the above method. Sharma and Koul reported $8.33 \mathrm{~mL}$ swelling factor for $P$. major in their study (12). The yield of mucilage extraction from $P$. major seeds was $20.9 \%$. The characteristics of evaluated tablets are shown in table 2 . 
Mucoadhesive tablets were prepared and analyzed as described above. The release rate of propranolol from matrix tablets increased, as the ratio of mucilage: Carbopol 934P increased in formulation F1 to F7 (Fig. 1). And results show the release rate decreases, as the amount of mucilage and carbopol increased in formulation F8-F13 (Fig. 2). The lowest release rate was with formulation F1 (Containing $10 \mathrm{mg} P$. major mucilage and $70 \mathrm{mg}$ Carbopol 934P) and the highest release rate was with formulation $\mathrm{F} 7$
(Containing $70 \mathrm{mg} P$. major mucilage and $10 \mathrm{mg}$ Carbopol 934P) and the percent of cumulative drug release after $2 \mathrm{hr}$ for F8F13 were 56.24, 45.2, 32.18, 73.98, 22.68, 12.31 respectively. The lowest release rate was observed with formulation F13 (Containing $160 \mathrm{mg}$ Carbopol 934P) and the highest was with F11 (Containing 40 mg Carbopol 934P). The significant difference was observed between formulations $(\mathrm{p}<0.001)$.

Table 2 Characteristics of propranolol hydrochloride tablets prepared with different ratios of $P$. major mucilage and Carbopol934P.

\begin{tabular}{ccccc}
\hline $\begin{array}{c}\text { Formulation } \\
\text { code }\end{array}$ & $\begin{array}{c}\text { Hardness*(Kg/cm }) \\
(\mathrm{n}=10)\end{array}$ & $\begin{array}{c}\text { Friability } \\
(\% \mathrm{w} / \mathrm{w}) \\
(\mathrm{n}=10)\end{array}$ & $\begin{array}{c}\text { Tensile } \\
\text { strength*(MN/m})\end{array}$ & $\begin{array}{c}\text { Assay }(\%) \\
(\mathrm{n}=20)\end{array}$ \\
\hline F1 & $3.65 \pm 0.16$ & $1.22 \pm 0.11$ & $0.43 \pm 0.05$ & $79.7 \pm 1.4$ \\
\hline F2 & $3.82 \pm 0.22$ & $1.03 \pm 0.05$ & $0.45 \pm 0.06$ & $80.1 \pm 1.5$ \\
\hline F3 & $4.10 \pm 0.19$ & $0.96 \pm 0.06$ & $0.52 \pm 0.06$ & $79.6 \pm 2.8$ \\
\hline F4 & $3.25 \pm 0.18$ & $1.02 \pm 0.04$ & $0.33 \pm 0.07$ & $80.2 \pm 2.1$ \\
\hline F5 & $3.48 \pm 0.29$ & $0.98 \pm 0.07$ & $0.39 \pm 0.05$ & $78.8 \pm 0.8$ \\
\hline F6 & $3.57 \pm 0.25$ & $0.91 \pm 0.05$ & $0.42 \pm 0.06$ & $79.5 \pm 0.7$ \\
\hline F7 & $4.75 \pm 0.29$ & $0.92 \pm 0.05$ & $0.58 \pm 0.07$ & $81.4 \pm 0.81$ \\
\hline F8 & $4.95 \pm 0.37$ & $0.86 \pm 0.06$ & $0.65 \pm 0.05$ & $78.0 \pm 1.2$ \\
\hline F9 & $5.57 \pm 0.35$ & $0.81 \pm 0.05$ & $0.74 \pm 0.06$ & $81.1 \pm 0.83$ \\
\hline F10 & $4.91 \pm 0.28$ & $0.91 \pm 0.05$ & $0.61 \pm 0.07$ & $78.9 \pm 1.7$ \\
\hline F11 & $5.23 \pm 0.32$ & $0.82 \pm 0.07$ & $0.68 \pm 0.06$ & $79.3 \pm 0.8$ \\
\hline F12 & $5.38 \pm 0.23$ & $0.86 \pm 0.08$ & $0.67 \pm 0.04$ & $80.2 \pm 1.3$ \\
\hline F13 & $4.98 \pm 0.34$ & $0.83 \pm 0.06$ & $0.72 \pm 0.05$ & $79.4 \pm 0.6$ \\
\hline
\end{tabular}

*Data are shown as mean $\pm \mathrm{SD}$ 


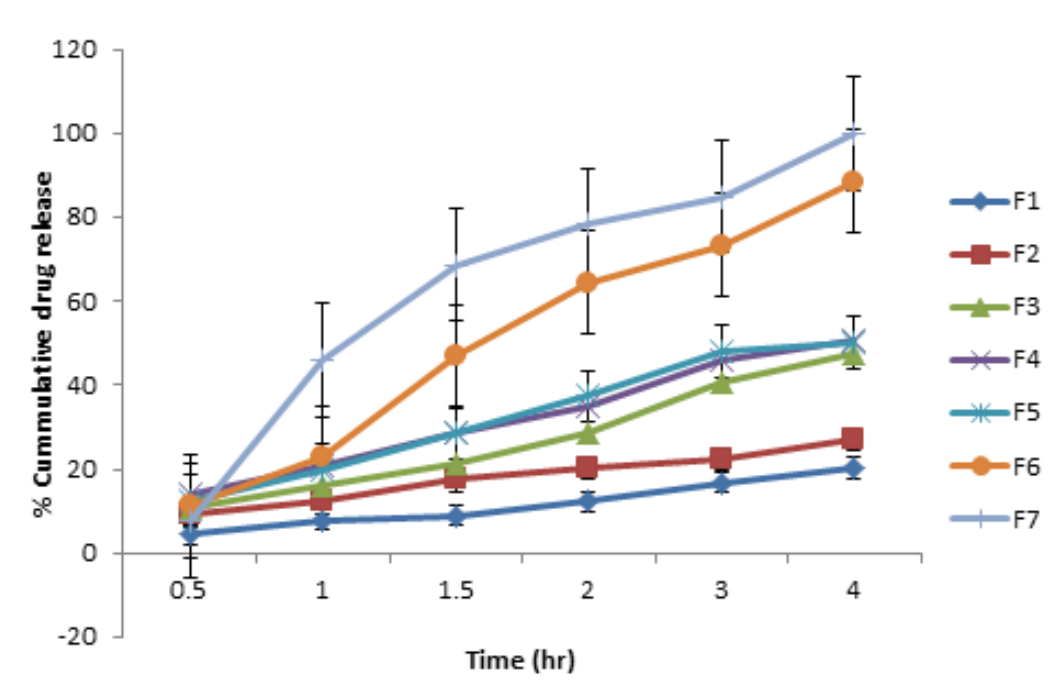

Figure 1 Comparison of release profile of propranolol hydrochloride from formulation F1-F7 $(\mathrm{n}=3)$.

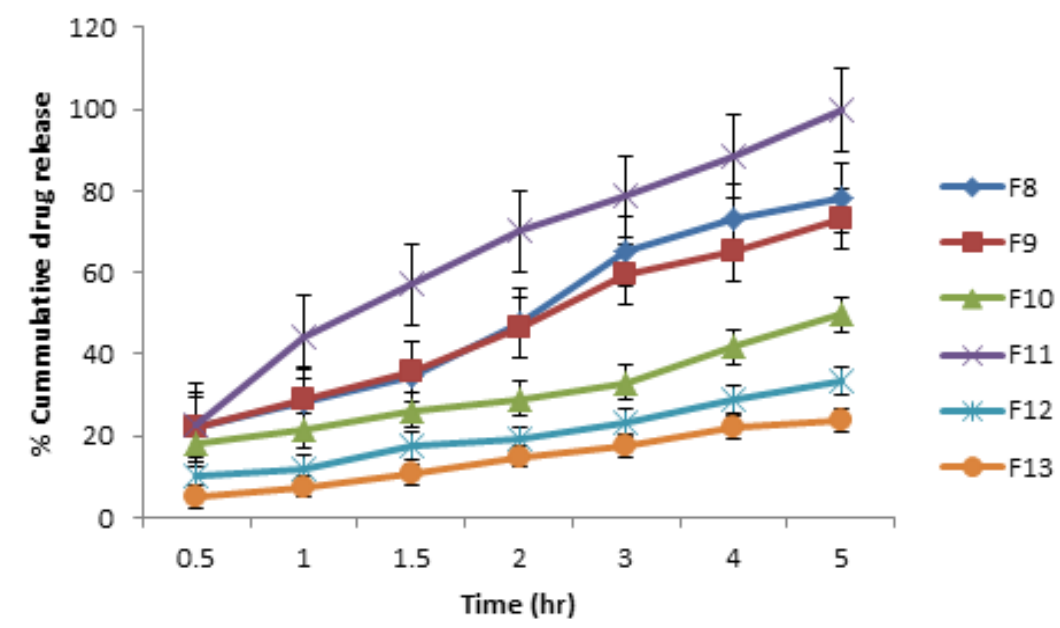

Figure 2 Comparison of release profile of propranolol hydrochloride from formulation F8-F13 ( $\mathrm{n}=3)$

Dissolution rate data were analyzed based on equations 2-5 and their results are listed in table3. The results showed that the amount and type of the polymer affect the release kinetic of propranolol from tablets, the highest correlation coefficient were achieved with the various models. 
Table 3 The kinetic release parameters for propranolol mucoadhesive tablets formulations

\begin{tabular}{|c|c|c|c|c|c|c|c|c|c|c|c|c|c|}
\hline \multirow{2}{*}{$\begin{array}{c}\text { Formul } \\
\text { ation } \\
\text { code }\end{array}$} & \multicolumn{3}{|c|}{ Zero-order model } & \multicolumn{3}{|c|}{ First-order model } & \multicolumn{3}{|c|}{ Higuchi model } & \multicolumn{4}{|c|}{ Peppas model } \\
\hline & $\begin{array}{c}\mathrm{K}_{0} \\
\% \text { min }\end{array}$ & $\mathrm{r}^{2}$ & ss & $\begin{array}{c}\mathrm{K}_{1} \\
1 / \mathrm{min}\end{array}$ & $\mathrm{r}^{2}$ & ss & $\mathrm{K}_{\mathrm{H}}$ & $\mathrm{r}^{2}$ & ss & $\begin{array}{c}\mathrm{K}_{\mathrm{p}} \% \mathrm{~m} \\
\text { in }\end{array}$ & $\mathrm{n}$ & $\mathrm{r}^{2}$ & ss \\
\hline $\mathrm{F} 1$ & 0.0006 & 0.9840 & 11174.2 & -0.0008 & 0.9916 & 6057.4 & 0.0180 & 0.9915 & 38045.5 & 0.0037 & 0.7317 & 0.9942 & 247.6 \\
\hline $\mathrm{F} 2$ & 0.0007 & 0.9767 & 24065.7 & -0.0010 & 0.9843 & 15075.4 & 0.0199 & 0.9865 & 1678.9 & 0.0143 & 0.5445 & 0.9895 & 241.0 \\
\hline F3 & 0.0012 & 0.9422 & 18732.6 & -0.0020 & 0.9797 & 5384.8 & 0.0336 & 0.9859 & 13427.0 & 0.0112 & 0.6661 & 0.9862 & 447.9 \\
\hline $\mathrm{F} 4$ & 0.0011 & 0.9307 & 24724.6 & -0.0021 & 0.9797 & 9808.2 & 0.0329 & 0.9866 & 1549.4 & 0.0186 & 0.6013 & 0.9890 & 202.6 \\
\hline F5 & 0.0013 & 0.9383 & 22472.4 & -0.0025 & 0.9512 & 5085.0 & 0.0359 & 0.9714 & 6260.3 & 0.0163 & 0.6274 & 0.9627 & 753.5 \\
\hline F6 & 0.0032 & 0.9383 & 22472.4 & -0.0025 & 0.9512 & 5085.0 & 0.0753 & 0.9685 & 104829 & 0.0014 & 1.2773 & 0.9761 & 262.5 \\
\hline F7 & 0.0038 & 0.8037 & 9718.2 & -0.0372 & 0.7381 & 622808 & 0.0853 & 0.8987 & 267147 & 0.000 & 2.5919 & 1.000 & 0.000 \\
\hline F8 & 0.0014 & 0.8816 & 36561.4 & -0.0046 & 0.9920 & 5342.3 & 0.404 & 0.9629 & 2177.9 & 0.0296 & 0.6154 & 0.9982 & 7.4021 \\
\hline F9 & 0.0014 & 0.9252 & 30572.9 & -0.0037 & 0.9909 & 5654.2 & 0.0401 & 0.9629 & 2177.9 & 0.0305 & 0.5653 & 0.9682 & 11.04 \\
\hline F10 & 0.0012 & 0.9777 & 25318.1 & -0.0023 & 0.9990 & 8388.2 & 0.0334 & 0.9972 & 846.0 & 0.0266 & 0.5295 & 0.9936 & 92.4625 \\
\hline F11 & 0.0033 & 0.9256 & 13794.9 & -0.0283 & 0.8097 & 20277.1 & 0.0727 & 0.9761 & 2712.8 & - & - & - & - \\
\hline F12 & 0.0010 & 0.9162 & 19318.2 & -0.0016 & 0.9468 & 8041.4 & 0.0292 & 0.9694 & 12964.4 & 0.0100 & 0.6630 & 0.9795 & 668.3 \\
\hline F13 & 0.005 & 0.9915 & 22530.7 & -0.0006 & 0.9963 & 17088.9 & 0.0131 & 0.9940 & 2406.9 & 0.00089 & 0.549 & 0.9948 & 117.5 \\
\hline
\end{tabular}

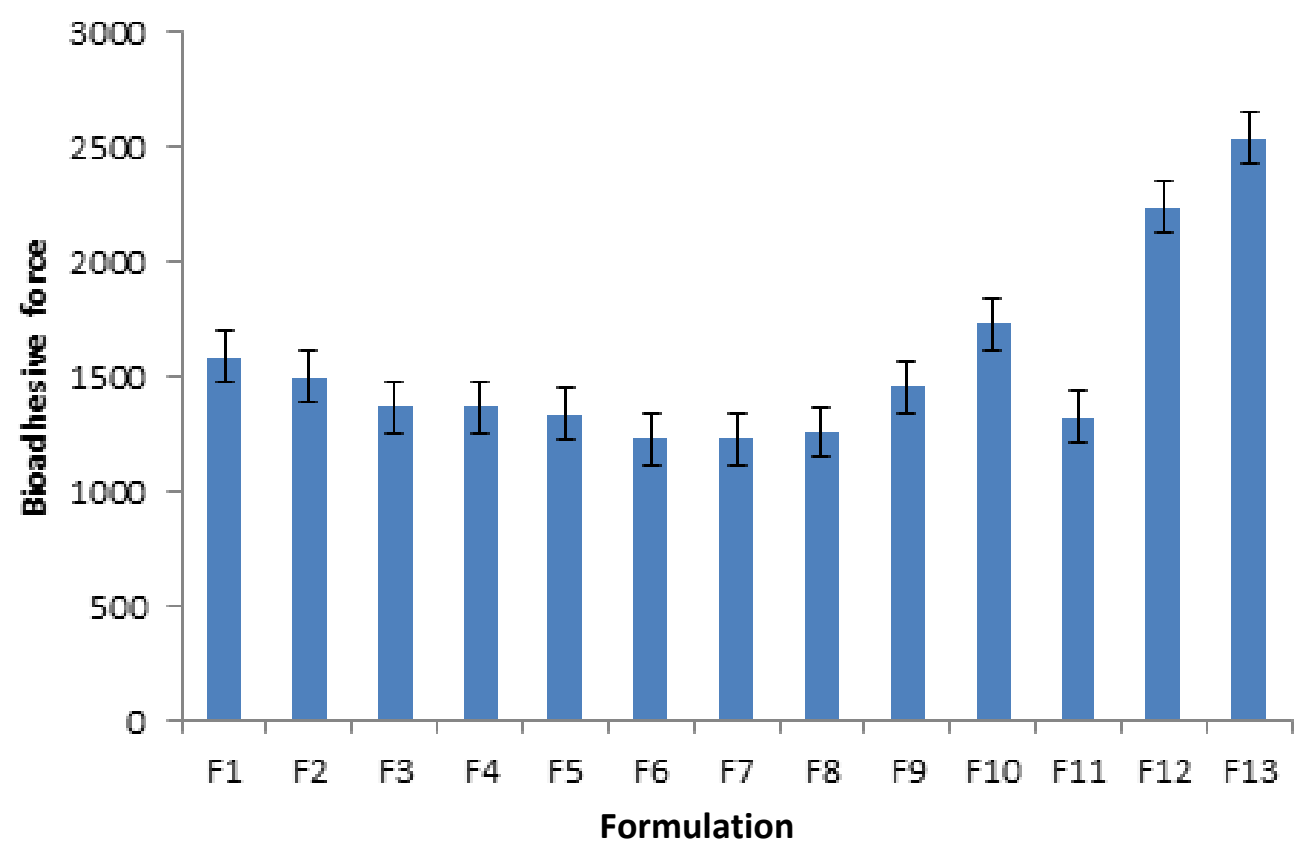

Figure 3 Comparison of bioadhesive force $\left(\mathrm{N} / \mathrm{m}^{2}\right)$ of formulations $\mathrm{F} 1-\mathrm{F} 13$ 
The bioadhesive strengths of mucoadhesive tablets are shown in Figure 3. The results show that all of the tablets have good bioadhesive strength, although the formulations with high ratio of Carbopol 934P: mucilage have higher bioadhesive strength but this difference were not significant between F1-F7 ( $p<$ 0.01). The results Also show that bioadhesive strength increased with increase in the amount of mucilage and carbopol 934P, so that the maximum bioadhesive strength was seen in F13.

The swelling of the polymers could be determined by water uptake of the tablet .The percent of swelling of the tablets were computed according to the method described previously. In this study the swelling of formulation F1-F7 were investigated (Fig. 4), The percent of polymers in each formulation was mentioned previously in table1. The F6 and F7 matrices disintegrated after 5 and 3 hours respectively. The hydration and erosion rates of tablets were measured, after the immersion of tablets in the test medium, to relate the observed phenomena of drug release with the rates or polymer hydration. The matrix erosion measured by the weight loss from matrix tablets immersed in dissolution media as a function of time (Fig. 5). Weight loss from the tablets was in constant progression until the end of test and the results show that the percent of erosion increased with the increase in the ratio of mucilage: carbopol 934P (Fig. 5), and also comparison between F9 \& F14 showed that the absence of active ingredient in F14 resulted lower percentage of erision (Fig. 6).

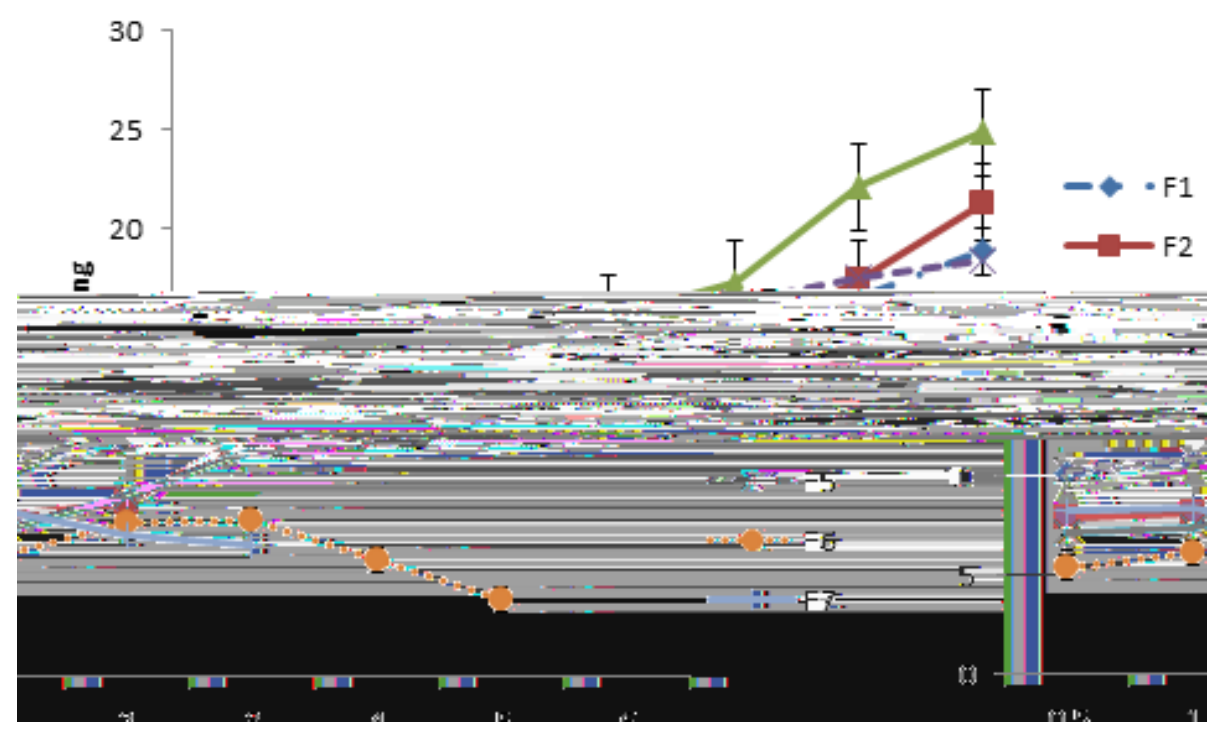

Figure 4 Comparison of swelling in F1-F7 formulations. 


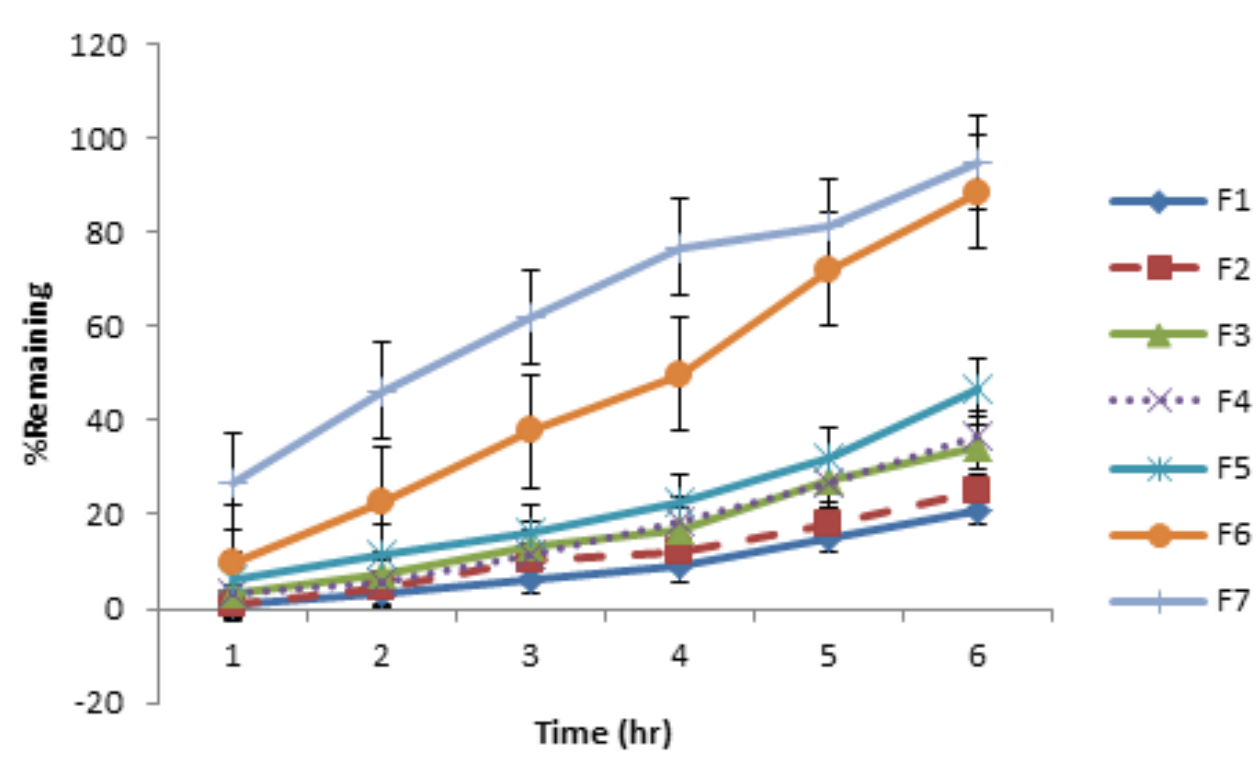

Figure 5 Comparison of erosion in F1-F7 formulations.

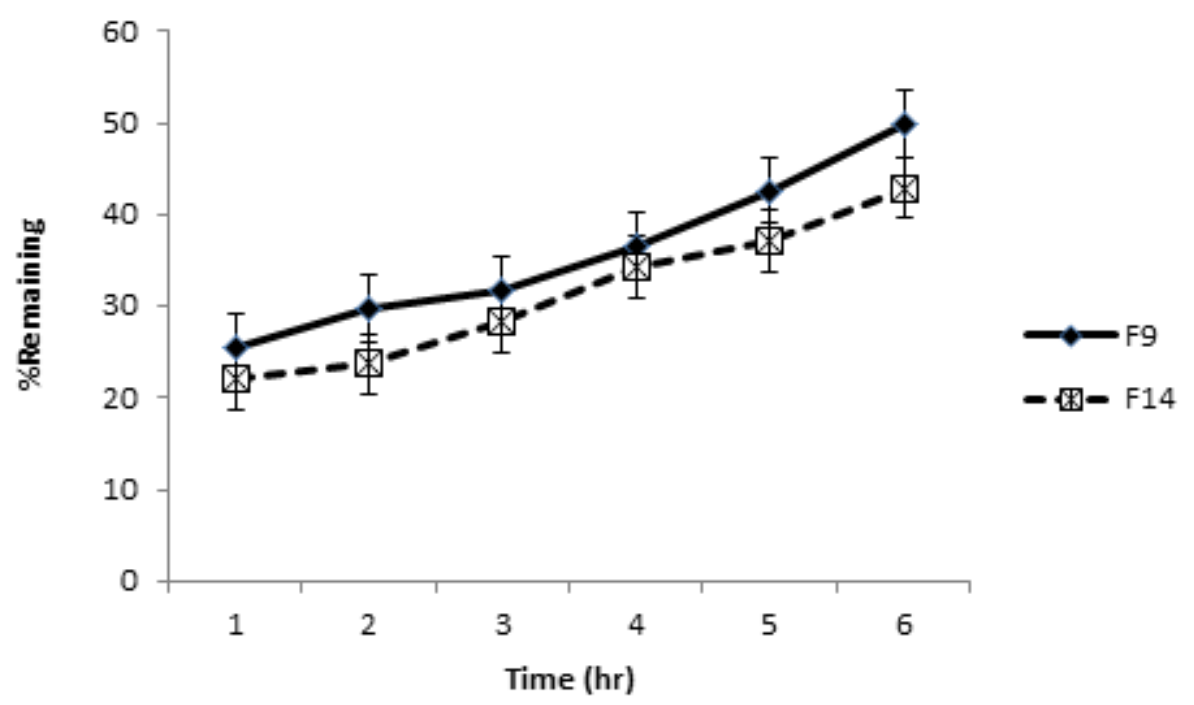

Figure 6 Comparison of erosion in F9 \& F14 formulations. 


\section{Discussion}

Values of the tensile strength and friability for all formulation are presented in Table 2.The values of hardness (from $3.6 \pm 0.16$ to $4.11 \pm 0.19 \mathrm{~kg} / \mathrm{cm}^{2}$ in $\mathrm{F} 1$ and $\mathrm{F} 3$ respectively) and tensile strength increased (from $0.43 \pm 0.5$ to $0.51 \pm 0.06 \mathrm{MN} / \mathrm{m}^{2}$ in $\mathrm{F} 1$ and F3 respectively) with increase in the concentration of mucilage while friability decreased with increase in mucilage concentration (from $1.22 \pm 0.11$ to $0.96 \pm$ $0.06 \%$ w/w in F1 and F3 respectively) for tablets prepared by direct compression .It is well known that polymers undergo plastic deformation, which subsequently leads to the formation of more solid bonds resulting in tablets with more resistance to fracture and abrasion (13).

When the polymer is "contaminated" by other materials such as the drug, the number of solid bonds between the particles decreased, leading to a decrease in the tensile strength of the tablets. The content uniformity test showed that the drug content was between $98.78-101.09 \%$.

The buccal route offers an attractive alternative for systemic drug delivery because of better patient compliance, ease of dosage form removal in emergencies, robustness, and good accessibility. Use of buccal mucosa for drug absorption was first attempted by Sobrero in 1847, and since then much research was done to deliver drugs through this route. Today research is more focused on the development of suitable delivery devices, permeation enhancement, and buccal delivery of drugs that undergo a first-pass effect, such as cardiovascular drugs, analgesics and peptides. In addition studies have been conducted on the development of controlled or slow release delivery systems for systemic or local therapy of diseases in the oral cavity (18).

Bio adhesive force means the force with which tablets binds to buccal mucous membranes. The bio adhesive forces of tablet are affected by the shape of the tablet, the swelling degree of the tablet and the nature of the tablet components (19).

Mucoadhesion defined as the state in which two materials, at least one biological in nature, are held together for an extended period of time by interfacial forces, the phenomena of bioadhesion occur by a complex mechanism. Till data, six theories have been proposed which can improve our understanding for the phenomena of adhesion and can also be extended to explain the mechanism of bio adhesion. The theories include: (a) the electronic theory, (b) the wetting theory, (c) the adsorption theory, (d) the diffusion theory, (e) the mechanical theory, (f) the cohesive theory. The electronic theory proposes transfer of electrons amongst the surfaces resulting in the formation of an electrical double layer thereby giving rise to attractive forces. The wetting theory postulates that if the contact angle of liquids on the substrate surface is lower, then there is a greater affinity for the liquid to the substrate surface. If two such substrate surface is brought in contact with each other in the presence of the liquid, the liquid may act as an adhesive amongst the substrate surface. The adsorption theory proposes the presence of intermolecular forces, viz. hydrogen binding, and Van der Waal's forces, for the adhesive interaction amongst the substrate surfaces. The diffusion theory assumes the diffusion of the polymer 
chains, present on the substrate surfaces, across the adhesive interface thereby forming a networked structure. The mechanical theory explains the diffusion of the liquid adhesives into the micro-cracks and irregularities present on the substrate surface thereby forming and interlocked structure which gives rise to adhesion. The cohesive theory proposes that the phenomena of bio adhesion are mainly due to the intermolecular interactions amongst like- molecules (20).

Formulations that contain Carbopol 934P had higher bio adhesive force in comparison to those had $P$. major mucilage, also the bio adhesion force increased as the amount of polymer increased, because of the increase in sites for bond formation. Formation of hydrogen bonds between the hydrophilic functional groups of the mucoadhesive polymers and the mucus layer is a prerequisite for extensive and longer mucoadhesion. The amount of the polymer also plays a significant role in the process of mucoadhesion. At lower amounts of the polymer chains, there is an inadequate and unsuitable interaction among the polymer and mucosal layer resulting in poor mucoadhesive properties (21).

$P$.major contain mucilaginous matter consisting of hydrophilic polysaccharides mainly in the seed coat, this polysaccharide has variable amounts of xylose, arabinose, galacturonic acid that swell in contact with water and form mucilage with high viscosity.

Formation of hydrogen bonds between the hydrophilic functional groups of the mucoadhesive polymers and the mucus layer or the mucosal surface is a prerequisite for extensive and longer mucoadhesion (22).

Vimal Kumar Yadav et al. studied Means of improving the bio adhesive properties of Drug Delivery Systems and the results shows that with the increase in the chain length of the polymers there is an increase in the mucoadhesive property of the polymer. Flexible Polymer chains help in the better penetration and entanglement of the polymer chains with that of mucosal layer thereby improving the bio adhesive property. The flexibility of the polymer chains is generally affected by the cross linking reaction and the hydration of the polymer network. The Higher the crosslinking density, the lower is the flexibility of the polymer chains (20).

Akbari et al evaluated the effect of fillers on the buccoadhesive propranolol tablets. It was investigated that by increasing in the amount of poly carbophil the bioadhesion force increased. Because the viscosity of the thin gel layer that is necessary for adhesion is increased by adding polycarbophil so the bio adhesion increases (16).

Comparison of the drug release profile of the tablets is shown in Fig. 1.The test was performed in phosphate buffer ( $\mathrm{pH}$ 6.8) and the samples studied at various times. Comparison showed that with increase in the amount of P.major mucilage and decrease in the amount of Carbopol 934P (F1 to F7) the drug release rate increases. and also for the formulation that have only one kind of polymer (F8 to F13) the drug release rate decreased with the increase in amount of polymer (Fig. 2)

Sabale et al studied the Calendula mucilage as a mucoadhesive substance and they demonstrated that with the increase in the 
amount of mucilage in the matrices the rate of drug release decreased (23).

Odeku and Fell investigated that the increase in the percentage of Khaya gum from 60 to $90 \%$ mucilage containing matrices resulted in a significant decrease in the release rate of paracetamol (13). Also in one study the researchers evaluate Aloe barbadensis miller mucilage as a component of control released matrices they understood that with an increase in the amount of mucilage in matrices the drug release rate decreases (24).

Swelling and erosion test was done in phosphate buffer (pH 6.8) are shown in fig. 4 $\& 5$. The data is not exhibiting a regular process in swelling in formulation $\mathrm{F} 1$ to $\mathrm{F} 3 \mathrm{p}$ $<0.01)$ we had an increase in the swelling percentage and from F4 to F7 we had a decrease in swelling complete disintegration was observed in F6 and F7 after 5hours and 3 hours respectively. High percentage of swelling in the F14 and F15 shows that the swelling in tablets that do not contain drug is higher than others. The friability was increased with the increase in amount of the mucilage and decrease in Carbopol 934P in formulation F1 to F7 ( $p$ < 0.05). Comparison of formulations F9 and F14 showed that erosion percentage in matrices with drug is higher than the matrices without it $(p<$ 0.005 ) because of the solubility of the drug that increases the erosion, and comparison of formulations F12 and F15 that contain Carbopo1934P shows that the percentage of erosion in matrices with drug is higher than the matrices with it $(\mathrm{p}<0.01)$

The adhesion increases with the degree of hydration until a point were over hydration leads to an abrupt drop in adhesive strength due to disentanglement at the polymer tissue interface (25). In hydrophilic polymeric matrix systems, the carrier on the surface of the matrix initially hydrates during dissolution to generate an outer viscous layer. This phase is then sequentially followed by matrix bulk hydration, swelling and erosion (26).

Adequate swelling behavior of a buccoadhesive system is an essential property for uniform and prolonged release of drug and effective mucoadhesion. Singh et al. studied the Mimosa pudica seed mucilage as a sustained release excipient in diclofenac sodium tablets and they understood as the amount of mucilage was increased in tablets percent swelling increased and percent erosion decreased (19).

Polymorphic changes of the drug may affect the dissolution rate and bioavailability. Bartolomei et al. reported that (R, S) propranolol hydrochloride existed in two crystalline forms, designated that $\alpha, \beta$.

According to the DSC test we did in our previous study $P$. major mucilage has a very broad endothermic peak at $95.2{ }^{\circ} \mathrm{C}$ the presence of this peak is because the loss of water from the carbohydrate polymer, and Propranolol thermograms showed an endothermic peak about $164.2 \pm 0.1{ }^{\circ} \mathrm{C}$, that is around its melting point. We tested the matrix tablet containing 1:1 of drugs: $P$. major mucilage ratio in both mixture tablet and physical mixture and it showed the same peak in this area which indicates no interaction between drug and mucilage during the formulation process (8). From this finding we understand the delayed dissolution rate of propranolol is not because of complex formation between the drug and P. major mucilage or changes in crystallinity of the drug. 


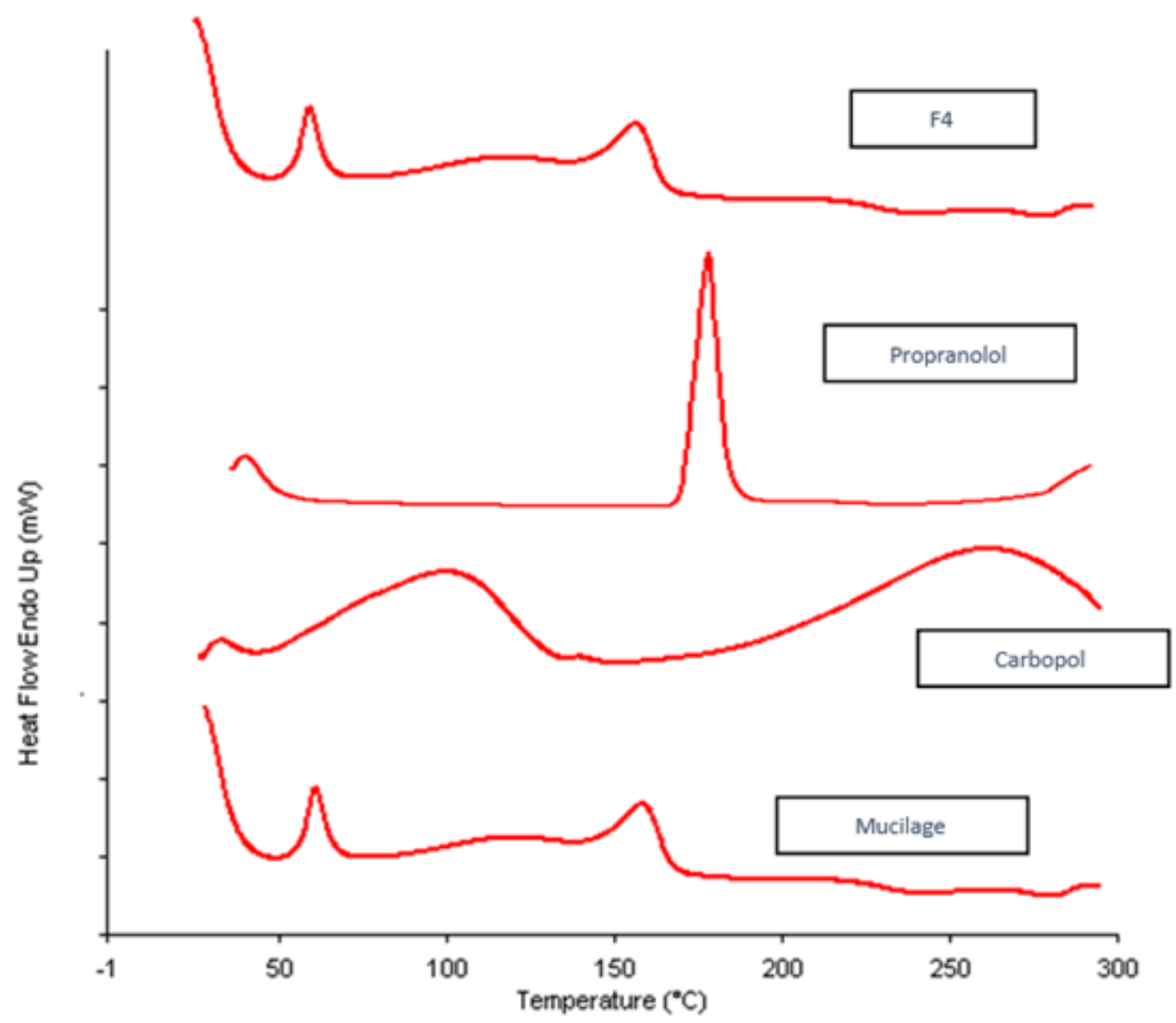

Figure 6 DSC thermograms of mucilage, Carbopol, Propranolol and formulation No. 4.

FT-IR spectral confirmed the above conclusion, $P$. major mucilage showed a broad band around $3437.51 / \mathrm{cm}$ corresponds to $\mathrm{OH}$ stretching, wave number $1742.91 / \mathrm{cm}$ depicts the stretching vibration of $\mathrm{C}=\mathrm{O}$, and $1040.1 \mathrm{1} / \mathrm{cm}$ depicts the stretching vibration of $\mathrm{C}-\mathrm{O}$ group which was a characteristics of a polysaccharide. and the test for propranolol hydrochloride showed a peak at $2964.91 / \mathrm{cm}$ due to the presence of a secondary amine group, peaks at 3280.6
$1 / \mathrm{cm}$ due to the secondary hydroxyl group. A peak at 1267.8 showed The presence of stretching bond of aryl alkyl ether, and a peak at $979.91 / \mathrm{cm}$ was due to $\alpha$ substituted naphthalene (8). The FT-IR spectrum of the tablets contain 1:1 ratio of drug and $P$. major mucilage showed the same peak in the same area, which indicates no physical or chemical interactions amongst the components of the formulation and compatibility of the drug with the $P$. major mucilage. 


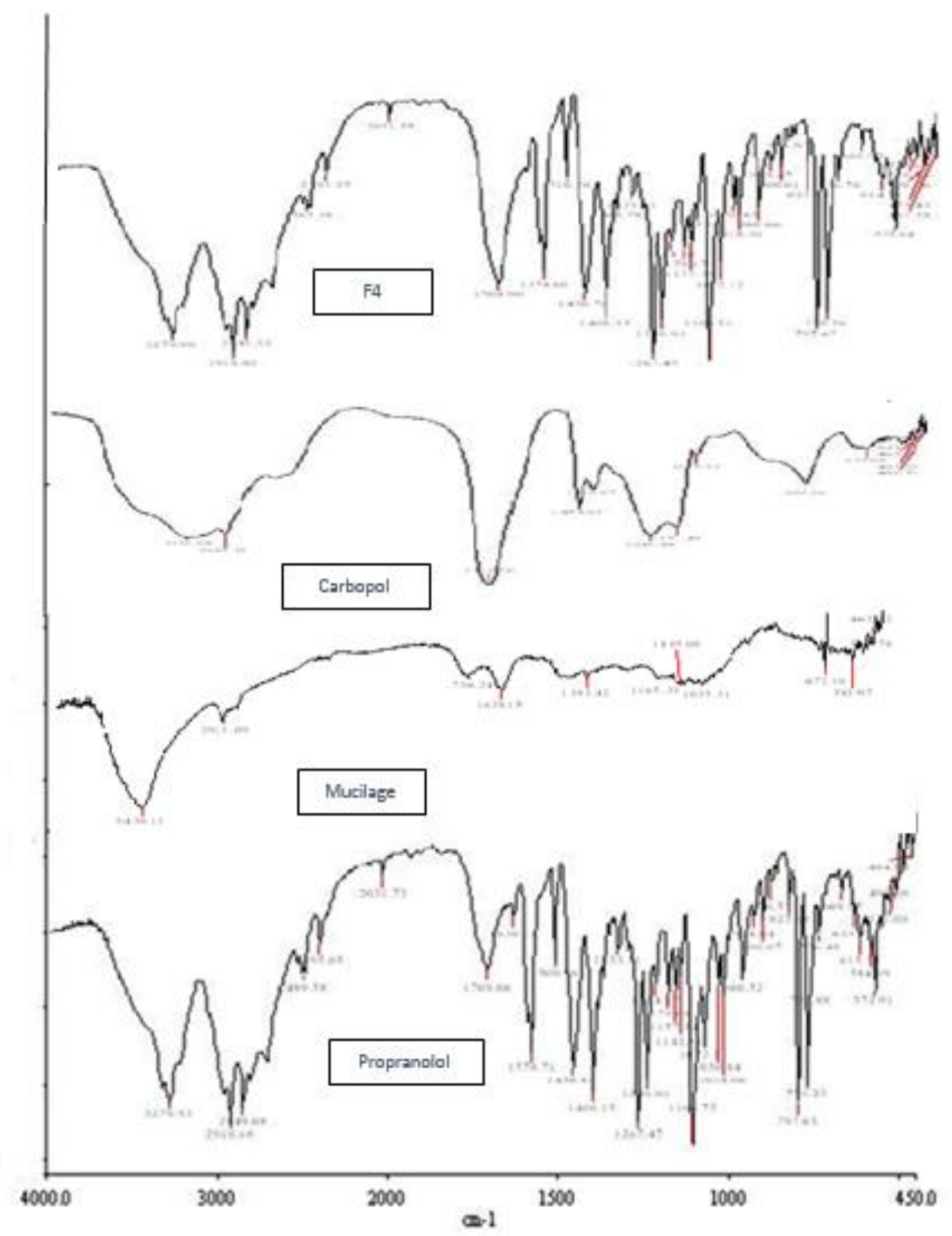

Figure 7 FTIR spectra of pure propranolol powder, mucilage, Carbopol and F4. 


\section{Conclusion}

The main aim of this work was to isolate $P$. major seed mucilage and evaluate this natural polymer as a component for release the drug from the buccal tablets and its adhesion to oral cavity mucosa together with Carbopol 934P. In conclusion the use of $P$. major mucilage in addition to carbopol can optimize the drug release in propranolol buccoadhesive tablets.

\section{References}

1. Bernkop-Schnurch A. Mucoadhesive systems in oral drug delivery. Drug Discov Today Technol 2005; 2:83-7.

2. Ahn JS, Choi HK, Cho CS. A novel mucoadhesive polymer prepared by template polymerization of acrylic acid in the presence of chitosan. Biomaterials 2001; 22: 923-8.

3. Hassan N, Khar RK, Ali M, Ali J. Development and evaluation of buccal bioadhesive tablet of an anti-emetic agent ondansetron. AAPS Pharm Sci Tech 2009;10:1085-92.

4. Roy SK. Prabhakar B. Bioadhesive polymeric platforms for transmucosal drug delivery systems - a review. Trop J Pharm Res 2010; 9: 91-104.

5. Chun MK, Cho CS, Choi HK. Mucoadhesive drug carrier based on interpolymer complex of poly(vinyl pyrrolidone) and poly(acrylic acid) prepared by template polymerization. J Control Release 2002;81:327-34.

6. Moghimipour E, Aghel N, Adelpour A. Formulation and Characterization of Oral Mucoadhesive Chlorhexidine Tablets Using Cordia myxa Mucilage. Jundishapur J Nat Pharm Prod 2012;7:129-33.

7. Saeedi M, Morteza-Semnani K, Sagheb-Doust M. Evaluation of Plantago major L. seed mucilage as a rate controlling matrix for sustained release of propranolol hydrochloride. Acta Pharm 2013;63: 99-114.

8. Sinha VR, Al-Azaki AA, Kumar RV. Novel Lannea Woodier gum matrices for controlled release of drugs. Carbohydr Polymers 2011; 83:1492-8.

9. Jani GK, Shah DP. Evaluation of mucilage of Hibiscus rosasinensis Linn as rate controlling

\section{Conflict of interest}

The authors declared no potential conflict of interest with respect to the authorship, and/or publication of this study. matrix for sustained release of diclofenac. Drug Dev Ind Pharm 2008;34:807-16.

10. Turel I, Ozbek H, Erten R, Oner AC, Cengiz N, Yilmaz O. Hepatoprotective and antiinflammatory activities of Plantago major L. Indian J Pharmacol 2009;41:120-4.

11. Samuelsen AB. The traditional uses, chemical constituents and biological activities of Plantago major L. A review. J Ethnopharmacol 2000;71:121.

12. British Pharmacopoeia 1993: HMSO, London, UK.

13. Odeku OA, Fell JT. Effects of the method of preparation on the compression, mechanical, and release properties of khaya gum matrices. Pharm Dev Technol 2006;11:435-41.

14. Peppas NA. Analysis of Fickian and non-Fickian drug release from polymers. Pharm Acta Helv 1985;60:110-1.

15. Akbari J, Nokhodchi A, Farid D, Adrangui M, Siahi-Shadbad MR, Saeedi M. Development and evaluation of buccoadhesive propranolol hydrochloride tablet formulations: effect of fillers. Farmaco 2004;59:155-61.

16. Pornsak Sriamornsaka B, Thirawonga N, Korkerd $\mathrm{K}$. Swelling, erosion and release behavior of alginate-based matrix tablets. Eur $\mathrm{J}$ Pharm Biopharm 2007;66:435-50.

17. Sharma PK, Koul AK. Mucilage in seeds of Plantago ovata and its wild allies. J Ethnopharmacol 1986;17:289-95.

18. Birudaraj R, Mahalingham R, Li X, Jasti B. Advances in buccal drug delivery. Crit Rev Ther Drug Carrier Syst 2005;22:295-330. 
19. Choi HG, Kim CK. Development of omeprazole buccal adhesive tablets with stability enhancement in human saliva. J Control Release 2000;68:397404.

20. Yadav VK, Kumar R, Yadav JS, Kumar B. Mucoadhesive polymers: means of improving the mucoadhesive properties of drug delivery system. J Chem Pharm Res 2010;2:418-32.

21. Roy S, Pal K, Anis A, Pramanik K, Prabhakar B. Polymers in mucoadhesive drug-delivery systems: A brief note. Designed Monomers Polymers 2009;12:483-95.

22. Alladi KK, Suram R, Bela M, Kiran S, Ramesh $\mathrm{V}$, Narendera Y. Formulation and characterization of Clarithromycin controlled release bioadhesive tablets. J Chem Pharm Res 2011;3:684-90.

23. Sabale V, Patel V, Paranjape A. Evaluation of Calendula mucilage as a mucoadhesive and controlled release component in buccal tablets. Res Pharm Sci 2014:9:39-48.
24. Saeedi M, Akbari J, Morteza-Semnani K, Azarashk A. Evaluation of Buccoadhesive Propranolol Hydrochloride Formulations Containing Plantago psyllium Seed Mucilage and Carbopol 934P. Iranian J Pharm Sci 2014;10:1-18.

25. Eouani C, Picerelle P, Prinderre P, Bourret E, Joachim J. In-vitro comparative study of buccal mucoadhesive performance of different polymeric films. Eur J Pharm Biopharm 2001;52:45-55.

26. Sinha Roy D, Rohera BD. Comparative evaluation of rate of hydration and matrix erosion of HEC and HPC and study of drug release from their matrices. Eur J Pharm Sci 2002;16:193-9. 